\title{
Elevated total homocysteine predicts in-hospital pneumonia and poor functional outcomes in acute ischemic stroke
}

\section{Shoujiang You}

Second Affiliated Hospital of Soochow University

\section{Lixuan Wang}

Second Affiliated Hospital of Soochow University

\section{Huaping Du}

the affiliated wujiang hospital of nantong universiry

\section{Danni Zheng}

The University of Sydney

\section{Chongke Zhong}

Soochow University

\section{Qiuyi Wu}

zhangjiagang first people hospital

\section{Chunqin Ding}

Second Affiliated Hospital of Soochow University

\section{Wanhua Wang}

kunshan first people hospital

Yongjun Cao ( $\square$ yongjuncao@126.com )

Chun-Feng Liu MD

Second Affiliated Hospital of Soochow University

\section{Research}

Keywords: acute ischemic stroke, total homocysteine, pneumonia, in-hospital outcomes

Posted Date: February 7th, 2020

DOI: https://doi.org/10.21203/rs.2.22904/v1

License: (a) (i) This work is licensed under a Creative Commons Attribution 4.0 International License.

Read Full License 


\section{Abstract}

Background The impact of elevated total homocysteine (tHcy) on functional outcomes and pneumonia after acute ischemic stroke (AIS) is still not well understood. We investigated the association between tHcy levels upon hospital admission and in-hospital short-term outcomes in AIS patients. Methods A total of 2,084 AIS patients enrolled from December 2013 to May 2014 across 22 hospitals in Suzhou city were included in the present study. We divided patients into 4 groups according to their level of admission tHcy: Q1 (<9.70 umol/L), Q2 (9.70-12.3 umol/L), Q3 (12.3-16.9 umol/L), and Q4 ( $\geq 16.9 \mathrm{umol} / \mathrm{L})$. Logistic regression models were used to estimate the effect of tHcy on the short-term outcomes, including inhospital pneumonia, all cause in-hospital mortality and poor outcome upon discharge (modified Rankin Scale score $\geq 3$ ) in AIS patients. Results During hospitalization, 332 patients $(15.9 \%)$ had pneumonia, 57 patients $(2.7 \%)$ died from all causes and $784(37.6 \%)$ patients experienced poor outcome at discharge. The risk of in-hospital pneumonia was significantly higher in patients with highest tHcy level (Q4) compared to those with lowest (Q1) (adjusted odds ratio [OR] 1.55; 95\% confidence interval [CI], 1.03-2.33; $P$-trend $=0.019)$. The highest thcy level (Q4) was associated with a 3.35 -fold and 1.50 -fold increase in the risk of in-hospital mortality (adjusted OR 3.35; 95\% Cl, 1.11-10.13; P -trend $=0.015$ ) and poor outcome upon discharge(adjusted OR 1.50; $95 \% \mathrm{Cl}, 1.06-2.12 ; \mathrm{P}$-trend $=0.044$ ) in comparison to Q1 after adjustment for potential covariates including pneumonia. Subgroup analyses further confirmed a significant association between higher tHcy levels and a high risk of short-term outcomes. Conclusions Having a high admission tHcy level was independently associated with in-hospital pneumonia, all cause in-hospital mortality and poor outcome upon discharge in AIS patients. Moreover, the association between higher tHcy and poor functional outcome was not modified by pneumonia.

\section{Introduction}

Stroke has been the leading cause of death and disability in China according to recent studies [1, 2]. Pneumonia is the most common infection after stroke and increases the risk of poor functional outcome among patients $[3,4]$. The identification of factors associated with in-hospital outcome, including pneumonia among stroke patients remains important [4], although the in-hospital mortality rate has not increased in the past decade in China [2].

Early epidemiologic studies had indicated that elevated serum total homocysteine (tHcy) is an important and independent risk factor for stroke $[5,6]$. Other studies had also investigated the prognostic effect of baseline elevated serum tHcy level on the clinical outcome in patients with acute ischemic stroke (AIS) [7-13]. However, the findings from those studies were inconsistent. The association between elevated serum tHcy and risk of poor functional outcome was noted in most [7-10], but not all studies [11-13] possibly due to differences in population sample size, sex and race. Recent studies had shown a combined effect of serum tHcy and high sensitivity C-reactive protein (hs-CRP) on clinical outcome among AIS patients suggesting tHcy may reflect underlying inflammation [14, 15]. Additionally, some studies found serum tHcy was an inflammation marker in spontaneous bacterial peritonitis and sepsis patients $[16,17]$. Therefore, we hypothesize that elevated serum tHcy at admission may be associated 
with pneumonia during hospital stay after AIS, which could contribute to poor functional outcome in patients.

Herein, in present study, we aimed to evaluate the association between elevated serum tHcy and inhospital pneumonia as well as in-hospital functional outcome in a large multicenter study of AIS patients from Suzhou, China.

\section{Methods}

\section{Study participants}

From December 2013 to May 2014, we recruited patients with AIS or transient ischemic attack (TIA) from 22 hospitals in Suzhou, China. Patients aged $\geq 18$ years with a clinical diagnosis of AIS or TIA were considered eligible. The methods for recruiting study participants have been detailed elsewhere $[18,19]$. Briefly, diagnosis of ischemic stroke was made according to World Health Organization-defined criteria based on patient history, clinical data, and neuroimaging results (computed tomography or magnetic resonance imaging). A team of investigators, including neurologists, reviewed the eligibility of study participants. Additional exclusion criteria were as follows: (1) diagnosis of TIA; and (2) time from onset to admission over 7 days. 3450 patients were potentially eligible for this analysis. Of these patients, 1366 were further excluded because of lack of serum tHcy concentration on admission (flowchart of participants selection; Fig. 1).

\section{Data Collection}

We collected baseline information, including patient demographics, vascular risk factors, stroke severity (National Institutes of Health Stroke Scale, NIHSS; Modified Rankin Scale score, mRS), medication use, imaging data and diagnosis-related information. Vascular risk factors included history of stroke, history of hypertension, history of diabetes mellitus, history of atrial fibrillation, history of coronary heart disease, current or previous smoking status, and alcohol consumption. Information on these factors were obtained by interviews with patients or their family members (if patients were not able to communicate). Current smoking status was defined as having smoked at least one cigarette per day for the previous year or more. Data on the amount and type of alcohol consumed during the past year was collected. Alcohol consumption was defined as having consumed at least one alcoholic drink per day during the past year. Hypertension was defined as having a systolic blood pressure (BP) $\geq 140 \mathrm{mmHg}$ and/or diastolic BP $\geq 90 \mathrm{mmHg}$ or use of antihypertensive medications. Diabetes mellitus was defined as having fasting glucose $\geq 7.0 \mathrm{mmol} / \mathrm{L}(126 \mathrm{mg} / \mathrm{dL})$, non-fasting glucose $\geq 11.1 \mathrm{mmol} / \mathrm{L}(200 \mathrm{mg} / \mathrm{dL})$ with classic symptoms of hyperglycemia or hyperglycemic crisis, use of glucose-lowering drugs. Atrial fibrillation was defined as having a history of atrial fibrillation, confirmed by $\geq 1$ electrocardiograms or the presence of arrhythmia during hospitalization. Pneumonia after AIS was diagnosed by treating physicians according to the criteria of US Center for Disease Control and Prevention for hospital-acquired pneumonia, based on 
clinical and laboratory test [20]. Blood samples were collected within 24 hours of hospital admission. Laboratory variables, including serum tHcy were assayed at local laboratories.

\section{Outcome Assessment}

The primary endpoint was in-hospital pneumonia and secondary outcomes were all-cause in-hospital mortality and poor functional outcome (defined as having a $m R S \geq 3$ ) at discharge.

\section{Statistical analysis}

Study participants were divided into four groups, based on quartiles of thcy levels at admission: Q1 (< $9.70 \mathrm{umol} / \mathrm{L}), \mathrm{Q} 2$ (9.70-12.3 umol/L), Q3 (12.3-16.9 umol/L), and Q4 ( $\geq 16.9 \mathrm{umol} / \mathrm{L})$. Continuous variables were expressed as mean \pm standard deviation (SD) or median (interquartile range [IQR]) and were compared using the analysis of variance or Wilcoxon rank-sum test. Categorical variables were expressed as frequency (\%) and were compared using the Chi-squared test.

The crude cumulative risks of in-hospital mortality for each quartile group of admission tHcy were shown in a Kaplan-Meier plot and compared using the log-rank test. Crude and multivariable logistic regression models were used to estimate the association between tHcy levels and the risk of in-hospital pneumonia, in-hospital mortality and poor functional outcome at hospital discharge. Odds ratios (ORs) and 95\% confidence interval (Cls) were calculated for each group with the lowest quartile (Q1) as reference. Potential confounders such as age, sex, systolic blood pressure, time from onset to admission, cigarette smoking status, alcohol drinking, history of hypertension, history of diabetes mellitus, history of coronary heart disease, history of atrial fibrillation, history of stroke, baseline NIHSS score $(\geq 4$ versus $<4)$ and estimated glomerular filtration rate (eGFR) levels were included in the multivariate models. For the evaluation of secondary outcomes including all-cause in-hospital mortality and poor functional outcome, pneumonia also was also adjusted for in the multivariable logistic regression models.

Subgroup analyses were conducted in multivariate adjusted models stratified by age ( $\geq 70$ years old versus $<70$ years old), sex, baseline systolic $B P(\geq 150 \mathrm{mmHg}$ versus $<150 \mathrm{mmHg}$ ), history of diabetes mellitus, and time from onset to hospital ( $\geq 24$ hours versus $<24$ hours). All $P$ values were two-tailed, with $<0.05$ considered statistically significant. All analyses were conducted using the SPSS Version 17.0 statistical software.

\section{Results}

There were 3450 AIS patients who had met the diagnostic criteria in our study, of which, 1366 (39.6\%) were excluded due to missing tHcy data. Baseline characteristics of enrolled and excluded patients are presented in Supplemental Table 1. Enrolled patients were more likely to be male, cigarette smoker, and tend to have shorter time from onset to hospitalization, but longer time of hospital stay. Other 
characteristics such as age, baseline NIHSS, baseline systolic BP, diastolic BP and the medical history were similar between two groups.

Complete data on conventional risk factors and tHcy levels at admission were available for 2,084 patients (1242 men and 842 women). The median age was 70 years (IQR 60-79), and the median NIHSS score was 4.0 (IQR 2.0-7.0). In comparison to participants with lower tHcy levels, those with higher tHcy levels were more likely to be older and male, cigarette smoker and consumer of alcohol and had comorbidities including hypertension and stroke. Patients with higher tHcy level were also more likely to have higher baseline systolic BP, and lower high-density lipoprotein cholesterol (HDL-C), fasting plasma glucose (FPG) and eGFR levels, and tended to have shorter time from onset to hospitalization (Table 1). 
Table 1

Baseline characteristics of 2,084 acute ischemic stroke patients according to serum thcy quartiles

Serum tHcy concentration, umol/l

\begin{tabular}{|c|c|c|c|c|c|}
\hline Characteristics $^{\text {a }}$ & $\begin{array}{l}\text { Q1 } \\
<9.70\end{array}$ & $\begin{array}{l}\text { Q2 } \\
9.70-12.3\end{array}$ & $\begin{array}{l}\text { Q3 } \\
12.3-16.9\end{array}$ & $\begin{array}{l}\text { Q4 } \\
\geq 16.9\end{array}$ & $\begin{array}{l}\mathrm{P}- \\
\text { value }\end{array}$ \\
\hline Number of subjects & 513 & 526 & 526 & 519 & \\
\hline \multicolumn{6}{|l|}{ Demographics } \\
\hline Age, y & $64.4 \pm 12.3$ & $67.5 \pm 11.9$ & $70.0 \pm 11.9$ & $70.9 \pm 13.7$ & $\begin{array}{l}< \\
0.001\end{array}$ \\
\hline Male sex & $222(43.3)$ & $306(58.2)$ & $344(65.4)$ & $370(71.3)$ & $\begin{array}{l}< \\
0.001\end{array}$ \\
\hline Cigarette smoking status & $71(13.8)$ & $116(22.1)$ & $126(24.0)$ & $135(26.0)$ & $\begin{array}{l}< \\
0.001\end{array}$ \\
\hline Alcohol consumption & $36(7.0)$ & $58(11.0)$ & $59(11.2)$ & 65 (12.5) & 0.025 \\
\hline \multicolumn{6}{|l|}{ Clinical features } \\
\hline $\begin{array}{l}\text { Time from onset to } \\
\text { hospital, } h\end{array}$ & $\begin{array}{l}24.0(6.0- \\
72.0)\end{array}$ & $\begin{array}{l}21.0(5.0- \\
48.0)\end{array}$ & $\begin{array}{l}24.0(5.0- \\
48.0)\end{array}$ & $\begin{array}{l}24.0(5.0- \\
48.0)\end{array}$ & 0.017 \\
\hline Hospital stay, day & $\begin{array}{l}10.0(8.0- \\
14.0)\end{array}$ & $\begin{array}{l}10.0(8.0- \\
14.0)\end{array}$ & $\begin{array}{l}10.0(8.0- \\
14.0)\end{array}$ & $\begin{array}{l}10.0(8.0- \\
14.0)\end{array}$ & 0.925 \\
\hline $\begin{array}{l}\text { Baseline systolic BP, mm } \\
\mathrm{Hg}\end{array}$ & $149.3 \pm 21.3$ & $151.4 \pm 22.1$ & $153.1 \pm 22.1$ & $154.8 \pm 24.7$ & 0.001 \\
\hline $\begin{array}{l}\text { Baseline diastolic BP, mm } \\
\mathrm{Hg}\end{array}$ & $85.3 \pm 12.2$ & $85.1 \pm 12.6$ & $85.4 \pm 13.3$ & $85.5 \pm 14.6$ & 0.976 \\
\hline $\mathrm{TG}, \mathrm{mmol} / \mathrm{L}$ & $1.2(0.9-1.8)$ & $1.2(0.9-1.7)$ & $1.2(0.8-1.6)$ & $1.2(0.9-1.6)$ & 0.061 \\
\hline $\mathrm{TC}, \mathrm{mmol} / \mathrm{L}$ & $4.6(4.0-5.4)$ & $4.6(3.9-5.3)$ & $4.5(3.9-5.2)$ & $4.5(3.8-5.2)$ & 0.099 \\
\hline LDL-C, mmol/L & $2.6(2.1-3.2)$ & $2.7(2.1-3.3)$ & $2.7(2.2-3.3)$ & $2.7(2.1-3.2)$ & 0.889 \\
\hline $\mathrm{HDL}-\mathrm{C}, \mathrm{mmol} / \mathrm{L}$ & $1.2(1.0-1.4)$ & $1.2(1.0-1.4)$ & $1.2(1.0-1.4)$ & $1.1(1.0-1.4)$ & 0.009 \\
\hline FPG, mmol/L & $5.9(5.2-7.5)$ & $6.0(5.1-7.6)$ & $5.6(5.1-6.9)$ & $5.6(4.9-6.6)$ & $\begin{array}{l}< \\
0.001\end{array}$ \\
\hline
\end{tabular}

*Continuous variables are expressed as mean \pm standard deviation or as median (interquartile range). Categorical variables are expressed as frequency (percent).

Abbreviations: BP, blood pressure; TG, triglycerides; TC, total cholesterol; LDL-C, low-density lipoprotein cholesterol; HDL-C, high-density lipoprotein cholesterol; FPG, fasting plasma glucose; eGFR, estimated glomerular filtration rate; mRS, modified Rankin Scale; NIHSS, National Institute of Health Stroke Scale; TACS, total anterior circulation syndrome; PACS, partial anterior circulation syndrome; POCS, posterior circulation syndrome; LACS, lacunar syndrome; Q, quartile. 


\section{Serum tHcy concentration, umol/l}

\begin{tabular}{|llllll|}
\hline eGFR, ml/min/1.73 ${ }^{2}$ & $112.1 \pm 32.1$ & $103.0 \pm 29.2$ & $94.2 \pm 30.3$ & $85.1 \pm 33.0$ & 0.001 \\
\hline mRS score & $2.0(2.0-3.0)$ & $2.0(2.0-3.0)$ & $2.0(2.0-4.0)$ & $3.0(2.0-4.0)$ & 0.110 \\
\hline NIHSS score & $4.0(2.0-6.0)$ & $4.0(2.0-6.0)$ & $4.0(2.0-7.0)$ & $4.0(2.0-8.0)$ & 0.087 \\
\hline Medical history & & & & & \\
\hline Hypertension & $373(72.7)$ & $403(76.6)$ & $414(78.7)$ & $422(81.3)$ & 0.009 \\
\hline Diabetes mellitus & $152(29.6)$ & $138(26.2)$ & $124(23.6)$ & $121(23.3)$ & 0.071 \\
\hline Coronary heart disease & $22(4.3)$ & $31(5.9)$ & $28(5.3)$ & $26(5.0)$ & 0.698 \\
\hline Atrial fibrillation & $69(13.5)$ & $78(14.8)$ & $81(15.4)$ & $89(17.1)$ & 0.421 \\
\hline Stroke & $96(18.7)$ & $109(20.7)$ & $129(24.5)$ & $139(26.8)$ & 0.008 \\
\hline Medication history & & & & & \\
\hline Antihypertensive therapy & $301(58.7)$ & $294(55.9)$ & $301(57.2)$ & $312(60.1)$ & 0.545 \\
\hline Antiplatelet therapy & $38(7.4)$ & $41(7.8)$ & $43(8.2)$ & $45(8.7)$ & 0.894 \\
\hline Anticoagulation therapy & $4(0.8)$ & $6(1.1)$ & $9(1.7)$ & $3(0.6)$ & 0.295 \\
\hline Antiglycemic therapy & $112(21.8)$ & $107(20.3)$ & $83(15.8)$ & $87(16.8)$ & 0.037 \\
\hline Statin therapy & $16(3.1)$ & $13(2.5)$ & $21(4.0)$ & $18(3.5)$ & 0.565 \\
\hline Thrombolysis treatment & $13(2.5)$ & $15(2.9)$ & $16(3.0)$ & $10(1.9)$ & 0.687 \\
\hline Stroke syndrome & & & & & 0.040 \\
\hline TACS & $42(8.2)$ & $51(9.7)$ & $40(7.6)$ & $69(13.3)$ & \\
\hline PACS & $242(47.2)$ & $256(48.7)$ & $278(52.9)$ & $255(49.1)$ & \\
\hline POCS & $138(26.9)$ & $121(23.0)$ & $125(23.8)$ & $110(21.2)$ & \\
\hline LACS & $91(17.7)$ & $98(18.6)$ & $83(15.8)$ & $85(16.4)$ & \\
\hline S & & & & & \\
\hline
\end{tabular}

*Continuous variables are expressed as mean \pm standard deviation or as median (interquartile range). Categorical variables are expressed as frequency (percent).

Abbreviations: BP, blood pressure; TG, triglycerides; TC, total cholesterol; LDL-C, low-density lipoprotein cholesterol; HDL-C, high-density lipoprotein cholesterol; FPG, fasting plasma glucose; eGFR, estimated glomerular filtration rate; mRS, modified Rankin Scale; NIHSS, National Institute of Health Stroke Scale; TACS, total anterior circulation syndrome; PACS, partial anterior circulation syndrome; POCS, posterior circulation syndrome; LACS, lacunar syndrome; Q, quartile.

During hospitalization, 332 patients $(15.9 \%)$ had a primary outcome of in-hospital pneumonia. In the multivariable model, the OR for pneumonia was significantly higher among study participants with 
admission tHcy in the highest quartile $(\geq 16.9 \mathrm{umol} / \mathrm{L})$ compared with those in the lowest quartile $(<9.70$ umol/L) (OR 1.55; 95\% Cl 1.03-2.33; P-trend = 0.019) after adjustment for age, sex, baseline NIHSS, and other potential covariates (Table 2). A significant association between admission tHcy levels and inhospital pneumonia was observed in most subgroups and there was no significant interaction between admission tHcy and subgroup variables (P-interaction $>0.05$ for all, Table 3 ).

Table 2

Odds ratios and $95 \%$ confidence intervals for in-hospital pneumonia according to quartiles of tHcy

\begin{tabular}{|c|c|c|c|c|c|c|c|}
\hline & & Unadjusted & & Model 1 & & Model 2 & \\
\hline & $\begin{array}{l}\text { Cases } \\
(\%)\end{array}$ & OR $(95 \% \mathrm{Cl})$ & $\begin{array}{l}\text { P- } \\
\text { trend }\end{array}$ & OR $(95 \% \mathrm{Cl})$ & $\begin{array}{l}\mathrm{P}- \\
\text { trend }\end{array}$ & OR $(95 \% \mathrm{Cl})$ & $\begin{array}{l}\text { P- } \\
\text { trend }\end{array}$ \\
\hline & & & $<.001$ & & 0.003 & & 0.019 \\
\hline$<9.70$ & $\begin{array}{l}58 \\
(11.3)\end{array}$ & $\begin{array}{l}1.00 \\
\text { (reference) }\end{array}$ & & $\begin{array}{l}1.00 \\
\text { (reference) }\end{array}$ & & $\begin{array}{l}1.00 \\
\text { (reference) }\end{array}$ & \\
\hline $\begin{array}{l}9.70- \\
12.3\end{array}$ & $\begin{array}{l}69 \\
(13.9)\end{array}$ & $\begin{array}{l}1.18(0.82- \\
1.72)\end{array}$ & & $\begin{array}{l}1.03(0.71- \\
1.51)\end{array}$ & & $\begin{array}{l}1.03(0.68- \\
1.55)\end{array}$ & \\
\hline $\begin{array}{l}12.3- \\
16.9\end{array}$ & $\begin{array}{l}91 \\
(17.3)\end{array}$ & $\begin{array}{l}1.64(1.15- \\
2.34)\end{array}$ & & $\begin{array}{l}1.29(0.89- \\
1.87)\end{array}$ & & $\begin{array}{l}1.22(0.82- \\
1.83)\end{array}$ & \\
\hline$\geq 16.9$ & $\begin{array}{l}114 \\
(22.0)\end{array}$ & $\begin{array}{l}2.21(1.57- \\
3.11)\end{array}$ & & $\begin{array}{l}1.63(1.13- \\
2.36)\end{array}$ & & $\begin{array}{l}1.55(1.03- \\
2.33)\end{array}$ & \\
\hline \multicolumn{8}{|c|}{ Model 1 , adjusted for age and sex; } \\
\hline \multicolumn{8}{|c|}{$\begin{array}{l}\text { Model 2, adjusted for age, sex, systolic blood pressure, time from onset to admission, cigarette } \\
\text { smoking status, alcohol drinking, history of hypertension, history of diabetes mellitus, history of } \\
\text { coronary heart disease, history of atrial fibrillation, history of stroke, baseline National Institutes of } \\
\text { Health Stroke Scale score (NIHSS } ₫ 4, \text { NIHSS } \geq 4 \text { )and eGFR levels. }\end{array}$} \\
\hline
\end{tabular}


Table 3

Odds ratios and $95 \%$ confidence intervals of in-hospital pneumonia according to quartiles of tHcy: subgroup analyses

\begin{tabular}{|c|c|c|c|c|c|c|}
\hline & \multicolumn{6}{|c|}{ Quartiles of serum tHcy } \\
\hline & Q1 & Q2 & Q3 & Q4 & $\begin{array}{l}\mathrm{P}- \\
\text { trend }\end{array}$ & $\begin{array}{l}\mathrm{P} \text { - } \\
\text { interaction }\end{array}$ \\
\hline Total participants & 1.00 & $\begin{array}{l}1.03(0.68- \\
1.55)\end{array}$ & $\begin{array}{l}1.22(0.82- \\
1.83)\end{array}$ & $\begin{array}{l}1.55(1.03- \\
2.33)\end{array}$ & 0.019 & \\
\hline \multicolumn{6}{|l|}{ Age, years } & 0.974 \\
\hline$\geq 70$ (median) & 1.00 & $\begin{array}{l}1.33(0.78- \\
2.26)\end{array}$ & $\begin{array}{l}1.27(0.75- \\
2.15)\end{array}$ & $\begin{array}{l}2.01(1.17- \\
3.44)\end{array}$ & 0.013 & \\
\hline$<70$ & 1.00 & $\begin{array}{l}0.66(0.34- \\
1.29)\end{array}$ & $\begin{array}{l}1.24(0.66- \\
2.33)\end{array}$ & $\begin{array}{l}1.11(0.58- \\
2.16)\end{array}$ & 0.403 & \\
\hline \multicolumn{6}{|l|}{ Sex } & 0.862 \\
\hline Female & 1.00 & $\begin{array}{l}0.93(0.53- \\
1.64)\end{array}$ & $\begin{array}{l}1.36(0.77- \\
2.40)\end{array}$ & $\begin{array}{l}1.51(0.82- \\
2.78)\end{array}$ & 0.110 & \\
\hline Male & 1.00 & $\begin{array}{l}1.12(0.61- \\
2.07)\end{array}$ & $\begin{array}{l}1.10(0.61- \\
2.01)\end{array}$ & $\begin{array}{l}1.55(0.86- \\
2.79)\end{array}$ & 0.110 & \\
\hline \multicolumn{6}{|c|}{ Baseline systolic BP, mmHg } & 0.091 \\
\hline$\geq 150$ (median) & 1.00 & $\begin{array}{l}1.01(0.58- \\
1.75)\end{array}$ & $\begin{array}{l}1.24(0.72- \\
2.15)\end{array}$ & $\begin{array}{l}2.18(1.26- \\
3.75)\end{array}$ & 0.002 & \\
\hline$<150$ & 1.00 & $\begin{array}{l}1.05(0.57- \\
1.95)\end{array}$ & $\begin{array}{l}1.21(0.66- \\
2.20)\end{array}$ & $\begin{array}{l}1.01(0.53- \\
1.94)\end{array}$ & 0.883 & \\
\hline \multicolumn{6}{|l|}{$\begin{array}{l}\text { History of diabetes } \\
\text { mellitus }\end{array}$} & 0.377 \\
\hline No & 1.00 & $\begin{array}{l}1.27(0.78- \\
2.06)\end{array}$ & $\begin{array}{l}1.40(0.86- \\
2.27)\end{array}$ & $\begin{array}{l}1.83(1.11- \\
3.01)\end{array}$ & 0.015 & \\
\hline Yes & 1.00 & $\begin{array}{l}0.60(0.27- \\
1.33)\end{array}$ & $\begin{array}{l}0.93(0.44- \\
1.97)\end{array}$ & $\begin{array}{l}1.22(0.57- \\
2.59)\end{array}$ & 0.447 & \\
\hline \multicolumn{6}{|l|}{$\begin{array}{l}\text { Time from onset to } \\
\text { hospital, h }\end{array}$} & 0.749 \\
\hline$\geq 24$ & 1.00 & $\begin{array}{l}0.81(0.43- \\
1.51)\end{array}$ & $\begin{array}{l}1.08(0.59- \\
1.96)\end{array}$ & $\begin{array}{l}1.44(0.80- \\
2.59)\end{array}$ & 0.107 & \\
\hline \multicolumn{7}{|c|}{ Model 1, adjusted for age and sex; } \\
\hline \multicolumn{7}{|c|}{$\begin{array}{l}\text { Model 2, adjusted for age, sex, systolic blood pressure, time from onset to admission, cigarette } \\
\text { smoking status, alcohol drinking, history of hypertension, history of diabetes mellitus, history of } \\
\text { coronary heart disease, history of atrial fibrillation, history of stroke, baseline National Institutes of } \\
\text { Health Stroke Scale score (NIHSS } ₫ 4 \text {, NIHSS } \geq 4 \text { )and eGFR levels. }\end{array}$} \\
\hline
\end{tabular}




\begin{tabular}{|c|c|c|c|c|c|}
\hline \multicolumn{6}{|c|}{ Quartiles of serum tHcy } \\
\hline$<24$ & 1.00 & $\begin{array}{l}1.23(0.70- \\
2.15)\end{array}$ & $\begin{array}{l}1.38(0.79- \\
2.40)\end{array}$ & $\begin{array}{l}1.80(1.00- \\
3.24)\end{array}$ & 0.046 \\
\hline \multicolumn{6}{|c|}{ Model 1 , adjusted for age and sex; } \\
\hline \multicolumn{6}{|c|}{$\begin{array}{l}\text { Model 2, adjusted for age, sex, systolic blood pressure, time from onset to admission, cigarette } \\
\text { smoking status, alcohol drinking, history of hypertension, history of diabetes mellitus, history of } \\
\text { coronary heart disease, history of atrial fibrillation, history of stroke, baseline National Institutes of } \\
\text { Health Stroke Scale score (NIHSS } \varangle 4 \text {, NIHSS } \geq 4 \text { )and eGFR levels. }\end{array}$} \\
\hline
\end{tabular}

Among 2,084 patients, 57 patients (2.7\%) had died from all causes during hospitalization. The cumulative incidence of all-cause mortality increased across the tHcy categories. (Log-rank $P=0.001$; Fig. 2) After adjustment for age, sex, baseline NIHSS, pneumonia and other potential covariates, study participants with admission tHcy in the highest quartile were significant associated with increased risk of in-hospital mortality compared with those in the lowest quartile (OR 3.35; 95\% $\mathrm{Cl} 1.11-10.13$; P-trend = 0.015) (Table 4). A significant association between admission tHcy levels and in-hospital mortality were observed in most subgroups and an interaction between admission tHcy and sex was noted ( $P$ interaction $=0.031)($ Table 5$)$. 
Table 4

Odds ratios and $95 \%$ confidence intervals for in-hospital outcome according to quartiles of tHcy

\begin{tabular}{|c|c|c|c|c|c|c|c|}
\hline & \multicolumn{3}{|c|}{ Unadjusted } & \multicolumn{2}{|l|}{ Model 1} & \multicolumn{2}{|l|}{ Model 2} \\
\hline & $\begin{array}{l}\text { Cases } \\
(\%)\end{array}$ & OR $(95 \% \mathrm{Cl})$ & $\begin{array}{l}\mathrm{P}- \\
\text { trend }\end{array}$ & OR $(95 \% \mathrm{Cl})$ & $\begin{array}{l}\mathrm{P}- \\
\text { trend }\end{array}$ & OR $(95 \% \mathrm{Cl})$ & $\begin{array}{l}\mathrm{P}- \\
\text { trend }\end{array}$ \\
\hline \multicolumn{2}{|l|}{ Death } & & $\begin{array}{l}< \\
0.001\end{array}$ & & 0.006 & & 0.015 \\
\hline$<9.70$ & $6(1.2)$ & \multicolumn{2}{|l|}{$\begin{array}{l}1.00 \\
\text { (reference) }\end{array}$} & \multicolumn{2}{|l|}{$\begin{array}{l}1.00 \\
\text { (reference) }\end{array}$} & \multicolumn{2}{|l|}{$\begin{array}{l}1.00 \\
\text { (reference) }\end{array}$} \\
\hline $\begin{array}{l}9.70- \\
12.3\end{array}$ & $\begin{array}{l}10 \\
(1.9)\end{array}$ & \multicolumn{2}{|l|}{$\begin{array}{l}1.64(0.59- \\
4.54)\end{array}$} & \multicolumn{2}{|l|}{$\begin{array}{l}1.42(0.51- \\
3.97)\end{array}$} & \multicolumn{2}{|l|}{$\begin{array}{l}1.46(0.46- \\
4.63)\end{array}$} \\
\hline $\begin{array}{l}12.3- \\
16.9\end{array}$ & $\begin{array}{l}15 \\
(2.9)\end{array}$ & \multicolumn{2}{|l|}{$\begin{array}{l}2.48(0.96- \\
6.44)\end{array}$} & \multicolumn{2}{|l|}{$\begin{array}{l}1.89(0.71- \\
5.01)\end{array}$} & \multicolumn{2}{|l|}{$\begin{array}{l}1.90(0.63- \\
5.74)\end{array}$} \\
\hline$\geq 16.9$ & $\begin{array}{l}26 \\
(5.0)\end{array}$ & \multicolumn{2}{|l|}{$\begin{array}{l}4.46(1.82- \\
10.92)\end{array}$} & \multicolumn{2}{|l|}{$\begin{array}{l}3.12(1.22- \\
7.98)\end{array}$} & \multicolumn{2}{|l|}{$\begin{array}{l}3.35(1.11- \\
10.13)\end{array}$} \\
\hline \multicolumn{3}{|c|}{$\begin{array}{l}\text { Poor functional } \\
\text { outcome }\end{array}$} & \multicolumn{2}{|l|}{$<.001$} & \multicolumn{2}{|l|}{0.002} & 0.044 \\
\hline$<9.70$ & $\begin{array}{l}160 \\
(31.2)\end{array}$ & \multicolumn{2}{|l|}{$\begin{array}{l}1.00 \\
\text { (reference) }\end{array}$} & \multicolumn{2}{|l|}{$\begin{array}{l}1.00 \\
\text { (reference) }\end{array}$} & \multicolumn{2}{|l|}{$\begin{array}{l}1.00 \\
\text { (reference) }\end{array}$} \\
\hline $\begin{array}{l}9.70- \\
12.3\end{array}$ & $\begin{array}{l}199 \\
(37.8)\end{array}$ & \multicolumn{2}{|l|}{$\begin{array}{l}1.34(1.04- \\
1.74)\end{array}$} & \multicolumn{2}{|l|}{$\begin{array}{l}1.33(1.02- \\
1.73)\end{array}$} & \multicolumn{2}{|l|}{$\begin{array}{l}1.45(1.05- \\
2.00)\end{array}$} \\
\hline $\begin{array}{l}12.3- \\
16.9\end{array}$ & $\begin{array}{l}206 \\
(39.2)\end{array}$ & \multicolumn{2}{|l|}{$\begin{array}{l}1.42(1.10- \\
1.84)\end{array}$} & \multicolumn{2}{|l|}{$\begin{array}{l}1.38(1.05- \\
1.80)\end{array}$} & \multicolumn{2}{|l|}{$\begin{array}{l}1.40(1.00- \\
1.96)\end{array}$} \\
\hline$\geq 16.9$ & $\begin{array}{l}219 \\
(42.2)\end{array}$ & \multicolumn{2}{|l|}{$\begin{array}{l}1.61(1.25- \\
2.08)\end{array}$} & \multicolumn{2}{|l|}{$\begin{array}{l}1.56 \\
2.05)\end{array}$} & \multicolumn{2}{|l|}{$\begin{array}{l}1.50(1.06- \\
2.12)\end{array}$} \\
\hline \multicolumn{8}{|c|}{ Model 1, adjusted for age and sex; } \\
\hline \multicolumn{8}{|c|}{$\begin{array}{l}\text { Model 2, adjusted for age, sex, systolic blood pressure, time from onset to admission, cigarette } \\
\text { smoking status, alcohol drinking, history of hypertension, history of diabetes mellitus, history of } \\
\text { coronary heart disease, history of atrial fibrillation, history of stroke, baseline National Institutes of } \\
\text { Health Stroke Scale score (NIHSS } ₫ 4 \text {, NIHSS } \geq 4 \text { ), eGFR levels and pneumonia. }\end{array}$} \\
\hline
\end{tabular}


Table 5

Odds ratios and $95 \%$ confidence intervals for in-hospital mortality according to quartiles of thcy: subgroup analyses

\begin{tabular}{|c|c|c|c|c|c|c|}
\hline & \multicolumn{6}{|c|}{ Quartiles of serum tHcy } \\
\hline & Q1 & Q2 & Q3 & Q4 & $\begin{array}{l}\text { P- } \\
\text { trend }\end{array}$ & $\begin{array}{l}\text { P- } \\
\text { interaction }\end{array}$ \\
\hline $\begin{array}{l}\text { Total } \\
\text { participants }\end{array}$ & 1.00 & $\begin{array}{l}1.46(0.46- \\
4.63)\end{array}$ & $\begin{array}{l}1.90(0.63- \\
5.74)\end{array}$ & $\begin{array}{l}3.35(1.11- \\
10.13)\end{array}$ & 0.015 & \\
\hline \multicolumn{6}{|l|}{ Age, years } & 0.865 \\
\hline$\geq 70$ (median) & 1.00 & $\begin{array}{l}1.03(0.26- \\
4.15)\end{array}$ & $\begin{array}{l}1.70(0.48- \\
6.05)\end{array}$ & $\begin{array}{l}3.01(0.84- \\
10.75)\end{array}$ & 0.036 & \\
\hline$<70$ & 1.00 & $\begin{array}{l}2.79(0.27- \\
28.52)\end{array}$ & $\begin{array}{l}2.57(0.24- \\
28.03)\end{array}$ & $\begin{array}{l}4.45(0.43- \\
46.15)\end{array}$ & 0.234 & \\
\hline \multicolumn{6}{|l|}{ Sex } & 0.031 \\
\hline Female & 1.00 & $\begin{array}{l}0.18(0.02- \\
1.71)\end{array}$ & $\begin{array}{l}1.48(0.37- \\
5.95)\end{array}$ & $\begin{array}{l}5.34(1.37- \\
20.88)\end{array}$ & 0.002 & \\
\hline Male & 1.00 & $\begin{array}{l}5.39(0.62- \\
46.68)\end{array}$ & $\begin{array}{l}2.84(0.32- \\
25.35)\end{array}$ & $\begin{array}{l}2.86(0.32- \\
26.22)\end{array}$ & 0.981 & \\
\hline \multicolumn{6}{|c|}{ Baseline systolic BP, mmHg } & 0.555 \\
\hline$\geq 150$ (median) & 1.00 & $\begin{array}{l}1.57(0.40- \\
6.19)\end{array}$ & $\begin{array}{l}1.87(0.50- \\
6.97)\end{array}$ & $\begin{array}{l}3.46(0.98- \\
12.32)\end{array}$ & 0.040 & \\
\hline$<150$ & 1.00 & $\begin{array}{l}1.07(0.09- \\
12.40)\end{array}$ & $\begin{array}{l}1.68(0.17- \\
16.83)\end{array}$ & $\begin{array}{l}2.51(0.24- \\
26.25)\end{array}$ & 0.252 & \\
\hline \multicolumn{6}{|l|}{$\begin{array}{l}\text { History of diabetes } \\
\text { mellitus }\end{array}$} & 0.590 \\
\hline No & 1.00 & $\begin{array}{l}1.56(0.38- \\
6.30)\end{array}$ & $\begin{array}{l}2.41(0.63- \\
9.29)\end{array}$ & $\begin{array}{l}3.40(0.86- \\
13.44)\end{array}$ & 0.038 & \\
\hline Yes & 1.00 & $\begin{array}{l}1.63(0.11- \\
24.06)\end{array}$ & $\begin{array}{l}0.76(0.05- \\
11.25)\end{array}$ & $\begin{array}{l}7.48(0.90- \\
62.27)\end{array}$ & 0.067 & \\
\hline \multicolumn{6}{|l|}{$\begin{array}{l}\text { Time from onset to } \\
\text { hospital, } h\end{array}$} & 0.795 \\
\hline$\geq 24$ & 1.00 & NA & NA & $\begin{array}{l}0.65(0.03- \\
15.70)\end{array}$ & 0.827 & \\
\hline \multicolumn{7}{|c|}{ Model 1, adjusted for age and sex; } \\
\hline $\begin{array}{l}\text { Model 2, adjusted } \\
\text { smoking status, al } \\
\text { coronary heart dis } \\
\text { Health Stroke Scal }\end{array}$ & rage, & ex, systolic b & $\begin{array}{l}\text { pressure, tim } \\
\text { yypertension, } \\
\text { ation, history } \\
\geq 4) \text {, eGFR leve }\end{array}$ & $\begin{array}{l}\text { n onset to ad } \\
\text { y of diabetes } \\
\text { oke, baseline } \\
\text { d pneumonia }\end{array}$ & $\begin{array}{l}\text { ion, cig } \\
\text { itus, his } \\
\text { onal Ins }\end{array}$ & $\begin{array}{l}\text { rette } \\
\text { pry of } \\
\text { itutes of }\end{array}$ \\
\hline
\end{tabular}




\begin{tabular}{|c|c|c|c|c|c|}
\hline \multicolumn{6}{|c|}{ Quartiles of serum tHcy } \\
\hline$<24$ & 1.00 & $\begin{array}{l}1.80(0.52- \\
6.26)\end{array}$ & $\begin{array}{l}2.37(0.71- \\
7.92)\end{array}$ & $\begin{array}{l}4.52(1.34- \\
15.28)\end{array}$ & 0.007 \\
\hline \multicolumn{6}{|c|}{ Model 1 , adjusted for age and sex; } \\
\hline \multicolumn{6}{|c|}{$\begin{array}{l}\text { Model 2, adjusted for age, sex, systolic blood pressure, time from onset to admission, cigarette } \\
\text { smoking status, alcohol drinking, history of hypertension, history of diabetes mellitus, history of } \\
\text { coronary heart disease, history of atrial fibrillation, history of stroke, baseline National Institutes of } \\
\text { Health Stroke Scale score (NIHSS } \varangle 4 \text {, NIHSS } \geq 4 \text { ), eGFR levels and pneumonia. }\end{array}$} \\
\hline
\end{tabular}

There were 784 patients (37.6\%) with poor functional outcome (mRS score $\geq 3$ ) at hospital discharge. After adjustment for age, sex, baseline NIHSS, pneumonia and other potential covariates, study participants with highest quartile of tHcy were significant associated with increased poor functional outcome risk compared with those in the lowest quartile (OR 1.50; $95 \% \mathrm{Cl} 1.06-2.12$; P-trend $=0.044$ ) (Table 4). Also, the significant association between admission tHcy levels and poor functional outcome at discharge was noted in most subgroups and an interaction between admission tHcy and baseline systolic BP was found $($-interaction $=0.038)($ Table 6$)$. 
Table 6

Odds ratios and $95 \%$ confidence intervals for poor functional outcome according to quartiles of tHcy: subgroup analyses

\begin{tabular}{|c|c|c|c|c|c|c|}
\hline & \multicolumn{6}{|c|}{ Quartiles of serum tHcy } \\
\hline & Q1 & Q2 & Q3 & Q4 & $\begin{array}{l}\text { P- } \\
\text { trend }\end{array}$ & $\begin{array}{l}\text { P- } \\
\text { interaction }\end{array}$ \\
\hline Total participants & 1.00 & $\begin{array}{l}1.45(1.05- \\
2.00)\end{array}$ & $\begin{array}{l}1.40(1.00- \\
1.96)\end{array}$ & $\begin{array}{l}1.50(1.06- \\
2.12)\end{array}$ & 0.044 & \\
\hline Age, years & & & & & & 0.188 \\
\hline$\geq 70$ (median) & 1.00 & $\begin{array}{l}1.16(0.70- \\
1.92)\end{array}$ & $\begin{array}{l}1.49(0.90- \\
2.46)\end{array}$ & $\begin{array}{l}1.94(1.14- \\
3.29)\end{array}$ & 0.007 & \\
\hline$<70$ & 1.00 & $\begin{array}{l}1.75(1.14- \\
2.69)\end{array}$ & $\begin{array}{l}1.21(0.75- \\
1.95)\end{array}$ & $\begin{array}{l}1.15(0.71- \\
1.86)\end{array}$ & 0.858 & \\
\hline Sex & & & & & & 0.078 \\
\hline Female & 1.00 & $\begin{array}{l}1.66(1.04- \\
2.63)\end{array}$ & $\begin{array}{l}1.37(0.83- \\
2.29)\end{array}$ & $\begin{array}{l}2.40(1.38- \\
4.18)\end{array}$ & 0.005 & \\
\hline Male & 1.00 & $\begin{array}{l}1.22(0.77- \\
1.94)\end{array}$ & $\begin{array}{l}1.29(0.81- \\
2.06)\end{array}$ & $\begin{array}{l}1.12(0.70- \\
1.79)\end{array}$ & 0.726 & \\
\hline Baseline systolic BF & $\mathrm{mmHc}$ & & & & & 0.038 \\
\hline$\geq 150$ (median) & 1.00 & $\begin{array}{l}1.88(1.21- \\
2.93)\end{array}$ & $\begin{array}{l}1.54(0.97- \\
2.45)\end{array}$ & $\begin{array}{l}2.13(1.32- \\
3.42)\end{array}$ & 0.009 & \\
\hline$<150$ & 1.00 & $\begin{array}{l}1.09(0.66- \\
1.77)\end{array}$ & $\begin{array}{l}1.34(0.81- \\
2.21)\end{array}$ & $\begin{array}{l}1.04(0.61- \\
1.77)\end{array}$ & 0.694 & \\
\hline $\begin{array}{l}\text { History of diabetes } \\
\text { mellitus }\end{array}$ & & & & & & 0.269 \\
\hline No & 1.00 & $\begin{array}{l}1.30(0.88- \\
1.91)\end{array}$ & $\begin{array}{l}1.24(0.83- \\
1.84)\end{array}$ & $\begin{array}{l}1.35(0.90- \\
2.04)\end{array}$ & 0.214 & \\
\hline Yes & 1.00 & $\begin{array}{l}1.83(0.99- \\
3.37)\end{array}$ & $\begin{array}{l}1.88(0.98- \\
3.60)\end{array}$ & $\begin{array}{l}2.20(1.12- \\
4.35)\end{array}$ & 0.026 & \\
\hline $\begin{array}{l}\text { Time from onset to } \\
\text { hospital, } h\end{array}$ & & & & & & 0.582 \\
\hline$\geq 24$ & 1.00 & $\begin{array}{l}1.65(1.02- \\
2.64)\end{array}$ & $\begin{array}{l}1.78(1.08- \\
2.92)\end{array}$ & $\begin{array}{l}1.70(1.04- \\
2.79)\end{array}$ & 0.048 & \\
\hline
\end{tabular}

Model 1, adjusted for age and sex;

Model 2, adjusted for age, sex, systolic blood pressure, time from onset to admission, cigarette smoking status, alcohol drinking, history of hypertension, history of diabetes mellitus, history of coronary heart disease, history of atrial fibrillation, history of stroke, baseline National Institutes of Health Stroke Scale score (NIHSS $\triangle 4$, NIHSS $\geq 4$ ), eGFR levels and pneumonia. 


\section{Quartiles of serum tHcy}

$\begin{array}{llllll}<24 & 1.00 & 1.27(0.81- & 1.13(0.70- & 1.32(0.80- & 0.418 \\ & & 2.01) & 1.81) & 2.18) & \end{array}$

Model 1, adjusted for age and sex;

Model 2, adjusted for age, sex, systolic blood pressure, time from onset to admission, cigarette smoking status, alcohol drinking, history of hypertension, history of diabetes mellitus, history of coronary heart disease, history of atrial fibrillation, history of stroke, baseline National Institutes of Health Stroke Scale score (NIHSS $₫ 4, \mathrm{NIHSS} \geq 4$ ), eGFR levels and pneumonia.

\section{Discussion}

The present study of over 2000 acute AIS patients had demonstrated an association between elevated tHcy and in-hospital outcomes. We found that having elevated tHcy at baseline was independently associated with the occurrence of in-hospital pneumonia, higher risk of in-hospital mortality as well as poor functional outcome at hospital discharge among AIS patients. Moreover, having pneumonia during hospitalization did not modify the effect of elevated thcy on patient outcome.

Total homocysteine is a sulfur-containing amino acid derived from methionine metabolism, and was an independent risk factor for stroke [5, 6]. Interestingly, several studies suggested elevated tHcy may reflect inflammation as other markers like hs-CRP [14-17]. A study of 977 acute AIS patients showed a combination of increased hs-CRP, tHcy, or white blood cell had a stronger predictive effect on 1-year poor outcome than individual elevated mediators [14]. Another study from China also found a concomitant effect of hs-CRP and tHcy on 1-year post-stroke depression [15]. Moreover, two studies suggested an association between early tHcy increase and poor outcome in patients with sepsis, and having elevated tHcy was a marker of spontaneous bacterial peritonitis in cirrhotic ascites patients $[16,17]$. In our study, we found acute AIS patients with elevated tHcy level had a 1.55-fold increased risk of in-hospital pneumonia. Our study is the first to reveal an association between elevated tHcy and the risk of pneumonia, consistent with previous reports of tHcy as a useful inflammatory marker.

Several studies had investigated the prognostic effect of elevated tHcy at admission on the functional outcome among patients with acute AIS. However, the findings from these studies were inconsistent [713,22]. A secondary analysis of the China Antihypertensive Trial in Acute Ischemic Stroke (CATIS) trial of 3309 AIS patients indicated higher Hcy was associated with worse acute AIS prognosis in women at 3month follow-up [7]. A study of 3799 AIS cases suggested that having elevated thcy levels could predict mortality, especially in stroke patients with the large-vessel atherosclerosis subtype during 48 months follow-up [8]. Other studies also noted that the higher level of tHcy may predict early neurological deterioration [9] and poor functional outcome at hospital discharge [10]. However, no significant association between higher tHcy and poor functional outcome was found in other studies [11-13, 22]. In a study which recruited 594 elderly AIS patients, elevated tHcy at admission was not shown to be a predictor of outcome at 3 months and 1 year [11]. A study of 775 AIS patients in Italy demonstrated 
higher tHcy was not associated with stroke severity nor outcome measured by the Barthel Index at 6 or 12 months [12]. In our study, we found elevated tHcy level was associated with a 3.35-fold and 1.50-fold increased risk of in-hospital mortality and poor functional outcome at hospital discharge respectively, and these associations were significant in different subgroups and in patients with pneumonia. Variations in sample size, population, definition of clinical outcomes and different statistic methods may have led to discrepant findings from previous studies.

In present study, we also found an interaction between high tHcy and gender on in-hospital mortality. The association between high thcy and increased risk of in-hospital mortality was observed only in females, but not males. Similar interesting phenomenon was seen in other studies [7, 23]. Zhong et al. using CATIS trial database had demonstrated elevated tHcy was associated with poor prognosis of acute AIS only in women [7] and a Swedish study also reported an association between higher tHcy and increased risk of 5-year mortality only in women [23]. The mechanism of gender-specific relationship between elevated tHcy and in-hospital mortality is unclear, partially due to early endothelial dysfunction caused by tHcy [24, 25] in women.

The exact mechanisms underlying the relationship between elevated tHcy levels and poor functional outcome after AIS is not yet fully understood. Several hypotheses have been proposed. First, previous studies showed a significant association between elevated tHcy and the presence of cerebral small vessel disease [26, 27], which is a known predictor of poor outcome and mortality after stroke [28, 29]. Second, animal studies indicated elevated tHcy significantly increased the blood-brain barrier dysfunction $[30,31]$, which may lead to poor functional outcome after stroke. Third, elevated tHcy was reported to increase oxidative stress [32] and exaggerates microglia activation and neuroinflammation after stroke [33].

Strengths of our study include having a relatively large number of patients from multiple centers and using sensitivity and subgroup analysis, which enhances the generalizability of the results. The present investigation is also the first to have found an independent association between elevated tHcy and pneumonia during hospitalization. However, there are also several potential limitations. First, a significant proportion of patients were excluded due to lack of homocysteine data, this may cause selection bias and contribute to a lower rate of in-hospital mortality compared to our previous studies. Secondly, in our study some patients had time from onset to admission which exceeded 24 hours, therefore, the levels of tHcy at admission might not accurately reflect the levels at stroke onset. However, our sensitivity analysis showed that the significance of the association remained when we restricted to patients with time from onset to admission $\leq 24$ hours. Thirdly, data on pre-stroke disability and Trial of Org 10172 in Acute Stroke Treatment (TOAST) classification, which may also influence in-hospital mortality. Moreover, the level of Vitamin B12 and folic acid, which are significant associated with tHcy were not collected. Finally, the follow-up period of our study is relatively short, thus we were unable to evaluate the combined longterm effect of tHcy on AIS outcomes and lacked information on exact cause of death.

\section{Conclusion}


Our results indicated that having a higher level of homocysteine at admission was independently associated with in-hospital pneumonia, in-hospital mortality and poor functional outcome at hospital discharge in acute ischemic stroke patients. The association between higher homocysteine and poor functional outcome remained significant considering the presence of pneumonia.

\section{Declarations}

\section{Acknowledgements}

We thank the study participants and their relatives and the clinical staff for their support and contribution to this study.

\section{Sources of Funding}

This work was supported in part by grants from the National Natural Science Foundation of China (81901198), Basic research of Suzhou Medical and health care (SYS201724), Suzhou Clinical Research Center of Neurological Disease (Szzx201503), Natural Science Foundation for Higher Education of Jiangsu Province of China (19KJB320004), the Second Affiliated Hospital of Soochow University Preponderant Clinic Discipline Group Project Funding (XKQ2015002). This work was also partly supported by Young Elite Scientists Sponsorship Program by CAST (2018QNRC001).

\section{Availability of data and materials}

The datasets during and/or analyzed during the current study are available from the corresponding author on reasonable request.

\section{Authors' contributions}

SJY, YJC, and CFL contributed to the concept and rationale for the study. SJY and LXW were responsible for the first draft; SJY, DZ and CKZ contributed statistical analyses. HPD, QYW, CQD and WHW performed the data collection; YJC and CFL for the first revision; All authors read and approved the final manuscript.

\section{Competing interests}

The authors declare no competing interests

\section{Consent for publication}

Not applicable

\section{Ethics approval and consent to participate}

The study protocol was approved by the Ethics Committee of the Second Affiliated Hospital of Soochow University, as well as ethical committees at the participating hospitals. Written consent was obtained from all study participants or their immediate family members. 


\section{References}

1. Wu S, Wu B, Liu M, Chen Z, Wang W, Anderson CS, et al. Stroke in china: Advances and challenges in epidemiology, prevention, and management. Lancet Neurol. 2019;18:394-405. 2. Wang W, Jiang B, Sun H, Ru X, Sun D, Wang L, et al. Prevalence, incidence, and mortality of stroke in china: Results from a nationwide population-based survey of 480687 adults. Circulation. 2017;135:759-71. 3. Finlayson 0, Kapral M, Hall R, Asllani E, Selchen D, Saposnik G. Risk factors, inpatient care, and outcomes of pneumonia after ischemic stroke. Neurology. 2011;77:1338-45. 4. Koennecke HC, Belz W, Berfelde D, Endres M, Fitzek S, Hamilton F, et al. Factors influencing in-hospital mortality and morbidity in patients treated on a stroke unit. Neurology. 2011;77:965-72. 5. Homocysteine and risk of ischemic heart disease and stroke: A meta-analysis. JAMA. 2002;288:2015-22. 6. Ji Y, Tan S, Xu Y, Chandra A, Shi C, Song B, et al. Vitamin b supplementation, homocysteine levels, and the risk of cerebrovascular disease: A metaanalysis. Neurology. 2013;81:1298-307. 7. Zhong C, Xu T, Xu T, Peng Y, Wang A, Wang J, et al. Plasma homocysteine and prognosis of acute ischemic stroke: A gender-specific analysis from catis randomized clinical trial. Mol neurobiol. 2017;54:2022-30. 8. Shi Z, Guan Y, Huo YR, Liu S, Zhang M, Lu H, et al. Elevated total homocysteine levels in acute ischemic stroke are associated with long-term mortality. Stroke. 2015;46:2419-25. 9. Kwon HM, Lee YS, Bae HJ, Kang DW. Homocysteine as a predictor of early neurological deterioration in acute ischemic stroke. Stroke. 2014;45:871-3. 10. Zhong C, Lv L, Liu C, Zhao L, Zhou M, Sun W, et al. High homocysteine and blood pressure related to poor outcome of acute ischemia stroke in chinese population. PloS one. 2014;9:e107498. 11. Wang W, Gao C, Yu C, Liu S, Hou D, Wang $Y$, et al. No association between elevated total homocysteine levels and functional outcome in elderly patients with acute cerebral infarction. Front Aging Neurosci. 2017;9:70. 12. Perini F, Galloni E, Bolgan I, Bader G, Ruffini R, Arzenton E, et al. Elevated plasma homocysteine in acute stroke was not associated with severity and outcome: Stronger association with small artery disease. Neurol Sci. 2005;26:310-8 13. Song IU, Kim YD, Kim JS, Lee KS, Chung SW. Can high-sensitivity c-reactive protein and plasma homocysteine levels independently predict the prognosis of patients with functional disability after first-ever ischemic stroke? Eur Neurol. 2010;64:304-10. 14. Gong X, Zou X, Liu L, Pu Y, Wang Y, Pan Y, et al. Prognostic value of inflammatory mediators in 1-year outcome of acute ischemic stroke with middle cerebral artery stenosis. Mediators Inflamm. 2013;2013:850714. 15. Cheng LS, Tu WJ, Shen Y, Zhang LJ, $\mathrm{Ji} \mathrm{K}$. Combination of high-sensitivity c-reactive protein and homocysteine predicts the post-stroke depression in patients with ischemic stroke. Mol Neurobiol. 2018;55:2952-8. 16. Kraja B, Babameto A, Burazeri G. Homocysteine: A new diagnostic marker for spontaneous bacterial peritonitis? Eur J Gastroenterol Hepatol. 2018;30:902-3. 17. Ploder M, Kurz K, Spittler A, Neurauter G, Roth E, Fuchs D. Early increase of plasma homocysteine in sepsis patients with poor outcome. Mol Med. 2010;16:498-504. 18. You S, Ou Z, Zhang W, Zheng D, Zhong C, Dong X, et al. Combined utility of white blood cell count and blood glucose for predicting in-hospital outcomes in acute ischemic stroke. J Neuroinflammation. 2019;16:37. 19. You S, Zheng D, Zhong C, Wang X, Tang W, Sheng L, et al. Prognostic significance of blood urea nitrogen in acute ischemic stroke. Circ J. 2018;82:572-8. 20. Garner JS, Jarvis WR, Emori TG, Horan TC, Hughes JM. Cdc definitions for nosocomial infections, 1988. Am J Infect Control. 1988;16:12840. 21. Huang X, Li Y, Li P, Li J, Bao H, Zhang Y, et al. Association between percent decline in serum total 
homocysteine and risk of first stroke. Neurology. 2017;89:2101-7. 22. Okubadejo NU, Oladipo OO, Adeyomoye AA, Awosanya GO, Danesi MA. Exploratory study of plasma total homocysteine and its relationship to short-term outcome in acute ischaemic stroke in nigerians. BMC Neurol. 2008;8:26. 23. Markaki I, Klironomos S, Kostulas K, Sjostrand C. Elevated plasma homocysteine upon ischemic stroke is associated with increased long-term mortality in women. PloS one. 2017;12:e0183571. 24. Cao C, Hu J, Dong Y, Zhan R, Li P, Su H, et al. Gender differences in the risk factors for endothelial dysfunction in chinese hypertensive patients: Homocysteine is an independent risk factor in females. PloS one. 2015;10:e0118686. 25. Moreau KL, Hildreth KL, Meditz AL, Deane KD, Kohrt WM. Endothelial function is impaired across the stages of the menopause transition in healthy women. $\mathrm{J}$ Clin Endocrinol Metab. 2012;97:4692-700. 26. Nam KW, Kwon HM, Jeong HY, Park JH, Kwon H, Jeong SM. Serum homocysteine level is related to cerebral small vessel disease in a healthy population. Neurology. 2019;92:e317-e325. 27. Piao X, Wu G, Yang P, Shen J, De A, Wu J, et al. Association between homocysteine and cerebral small vessel disease: A meta-analysis. J Stroke Cerebrovasc Dis.2018;27:2423-30. 28. Kim BJ, Lee SH.

Prognostic impact of cerebral small vessel disease on stroke outcome. J Stroke. 2015;17:101-10. 29. Association between brain imaging signs, early and late outcomes, and response to intravenous alteplase after acute ischaemic stroke in the third international stroke trial (ist-3): Secondary analysis of a randomised controlled trial. Lancet Neurol. 2015;14:485-96. 30. Kamath AF, Chauhan AK, Kisucka J, Dole VS, Loscalzo J, Handy DE, et al. Elevated levels of homocysteine compromise blood-brain barrier integrity in mice. Blood. 2006;107:591-3. 31. Beard RS, Jr., Reynolds JJ, Bearden SE. Hyperhomocysteinemia increases permeability of the blood-brain barrier by nmda receptor-dependent regulation of adherens and tight junctions. Blood. 2011;118:2007-14. 32. Kanani PM, Sinkey CA, Browning RL, Allaman M, Knapp HR, Haynes WG. Role of oxidant stress in endothelial dysfunction produced by experimental

hyperhomocyst(e)inemia in humans. Circulation. 1999;100:1161-8. 33. Chen S, Dong Z, Cheng M, Zhao Y, Wang $\mathrm{M}$, Sai $\mathrm{N}$, et al. Homocysteine exaggerates microglia activation and neuroinflammation through microglia localized stat3 overactivation following ischemic stroke. J Neuroinflammation. 2017;14:187.

\section{Figures}




\section{All patients with acute ischemic stroke and TIA $(n=3720)$}

1. Finally diagnosis with TIA $(n=206)$

2. Time from onset to admission more than 7 days $(n=64)$

\section{AIS patients}

\begin{tabular}{|c|}
\hline \multicolumn{1}{|c|}{} \\
\hline Missing serum \\
tHcy measurement \\
data $(\mathrm{n}=1366)$ \\
\hline$\downarrow$ \\
\hline Patients eligible for \\
Analysis $(\mathrm{n}=2084)$ \\
\hline
\end{tabular}

Figure 1

Patient flowchart. 


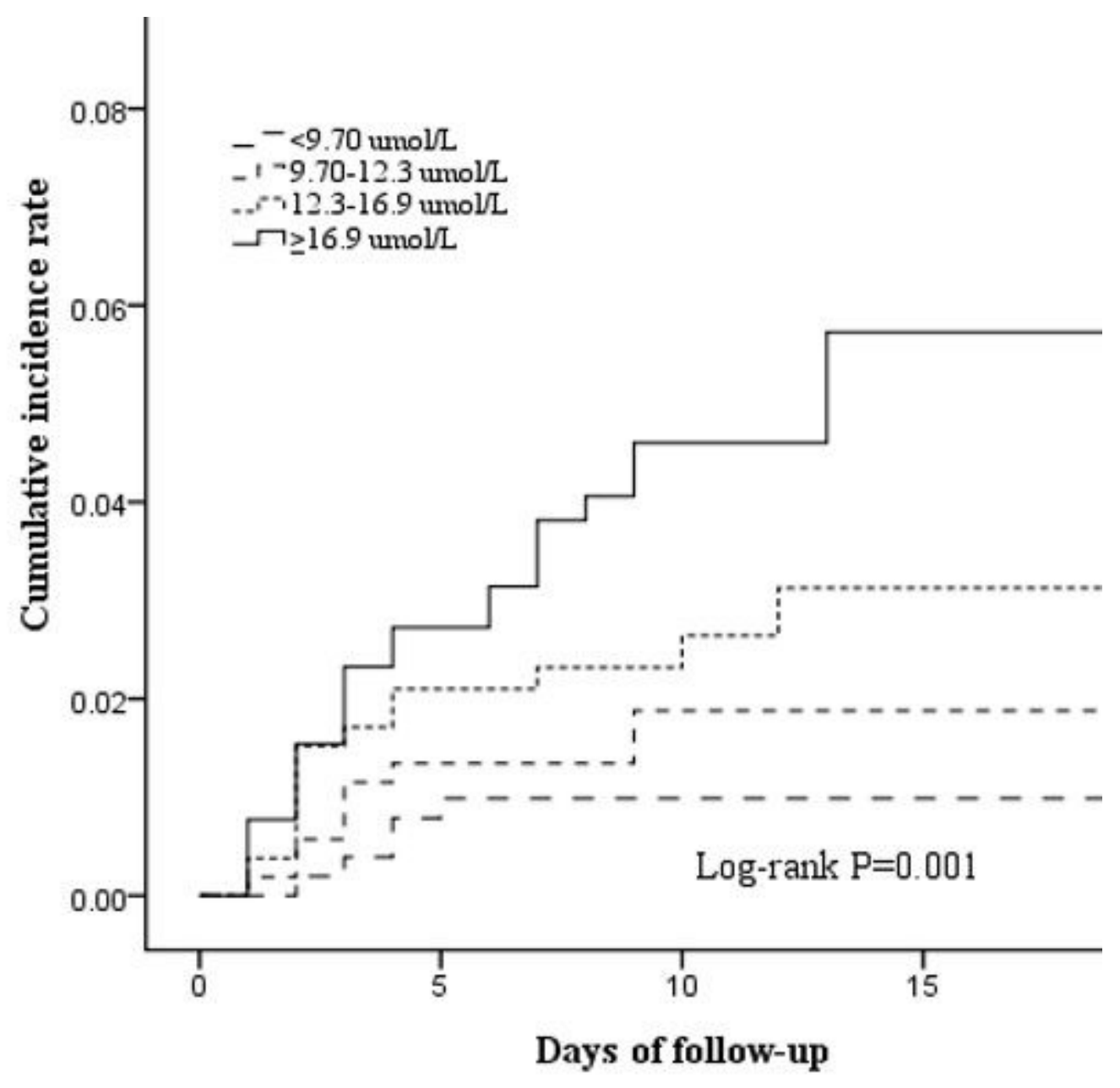

Figure 2

Cumulative incidence curves of in-hospital mortality by tHcy level.

\section{Supplementary Files}

This is a list of supplementary files associated with this preprint. Click to download.

- Supplementary131.doc 